\title{
S737F is a new CFTR mutation typical of patients originally from the Tuscany region in Italy
}

Vito Terlizzi ${ }^{*}$, Antonella Miriam Di Lullo ${ }^{2,3,4}$, Marika Comegna ${ }^{2,3}$, Claudia Centrone $^{5}$, Elisabetta Pelo ${ }^{5}$, Giuseppe Castaldo ${ }^{2,3}$, Valeria Raia ${ }^{6}$ and Cesare Braggion ${ }^{1}$

\begin{abstract}
Background: An increasing number of patients have been described as having a number of Cystic Fibrosis Transmembrane conductance Regulator (CFTR) variants for which it lacks a clear genotype-phenotype correlation. We assesses the clinical features of patients bearing the S737F (p.Ser737Phe) CFTR missense variant and evaluated the residual function of CFTR protein on nasal epithelial cells (NEC).

Methods: A retrospective database was performed from individuals homozygous or compound heterozygous for the S737F variant followed in the Cystic Fibrosis (CF) Centre of Florence. We performed a nasal brushing in cooperating patients and compared the results with those of patients followed in the pediatric CF Centre of Naples.

Results: 9/295 (3\%) subjects carrying at least S737F CFTR variant on one allele were identified. Patients were diagnosed in $7 / 9$ cases by newborn screening and in two cases for dehydration with hypochloremic metabolic alkalosis; at diagnosis sweat chloride levels (SCL) were in the pathological range in only one case. After a mean follow up of 8,6 years (range 0,5-15,8), SCL were in the pathological range in 8/9 cases (mean age at CF diagnosis: 1,5 years), all patients were pancreatic sufficiency and respiratory function was normal. The gating activity on NEC was 15 . $6 \%$ and $12.7 \%$ in two patients compound heterozygous for W1282X and DelE22_24, while it was ranged between $6,2 \%$ and $9,8 \%$ in CF patients.
\end{abstract}

Conclusions: S737F is a CFTR mutation associated to hypochloremic alkalosis in childhood, mild CF phenotype in teenage years and a residual function of CFTR protein.

Keywords: Cystic fibrosis, CFTR, Nasal brushing, Functional analysis, Gating, Genotype-phenotype correlation, Tuscany region, CFSPID, CRMS

\section{Background}

Cystic fibrosis (CF) is the most common life-threatening autosomal recessive disease in the US, affecting approximately 1 in 4000 newborns [1]. The prevalence in Europe is 0.737 per 10,000 and the incidence in Italy is estimated at 1/4,238 live [2]. It is caused by mutations of the CF Transmembrane conductance Regulator gene (CFTR) that encodes the cAMP-regulated chloride channel. About 2000 variants were described in the CFTR

\footnotetext{
* Correspondence: terlizzivito@libero.it

${ }^{1}$ Centro Regionale Toscano per la Fibrosi Cistica, Azienda

Ospedaliero-Universitaria Meyer, Via Gaetano Pieraccini 24, 50141 Florence,

Italy

Full list of author information is available at the end of the article
}

gene so far [3]. CFTR mutations can be classified into 6 classes according to the mechanism by which they impair CFTR protein synthesis, trafficking or function: class I mutations cause absent CFTR production, class II mutations affect intracellular processing of CFTR and class III mutations lead to defective regulation of the CFTR channel at the apical plasma membrane; classes IV, V and VI are associated to some degree of residual function $[3,4]$.

Although CFTR functions mainly as a chloride channel, it has many other regulatory roles, including inhibition of sodium transport through the epithelial sodium channel, regulation of the outwardly rectifying chloride channel, regulation of ATP channels, regulation 
of intracellular vesicle transport, acidification of intracellular organelles, and inhibition of endogenous calciumactivated chloride channels. CFTR is also involved in bicarbonate-chloride exchange. There are several hypotheses regarding how CFTR dysfunction leads to the phenotypic disease. One of the major is the lowvolume hypothesis postulating that the loss of inhibition of epithelial sodium channels, because of CFTR dysfunction, leads to excess sodium and water reabsorption, resulting in dehydration of airway surface materials [5].

Newborn screening (NBS) offers the opportunity for early diagnosis and improved outcome in patients with CF [6-8]. There continues to be considerable variability in structure of NBS programs [9]. NBS is done by the measurement of immunoreactive trypsinogen (IRT) in blood spots taken from newborn infants at day 3. Infants who have a high IRT concentration (>99th centile) undergo further assessment via a repeat IRT, 3 weeks later (IRT/IRT2), or by analysis of the initial blood spot for a specified group of CFTR mutations (IRT/DNA). A sweat test must still be done to confirm the diagnosis in infants with a positive NBS result. The sweat test remains the most readily available and clinically useful way of making the diagnosis of CF, provided it is done according to strict guidelines, with pilocarpine iontophoresis and a quantitative determination of chloride concentration $[1,10,11]$. A diagnosis of CF can be made in a patient with a positive NBS result and a sweat chloride concentrations greater than $60 \mathrm{mmol} / \mathrm{L}[1,10]$.

The CF phenotype includes multi-organ involvement with predominant severe respiratory disease, pancreatic insufficiency (PI) and male infertility [5]. In the last years an increasing number of patients have been described as having CFTR-related disorder (CFTR-RD) [12]. These cases are generally characterized by a later onset of symptoms such as pancreatitis, [13] disseminated bronchiectasis, congenital bilateral absence of vas deferens (CBAVD) associated to normal or borderline sweat chloride concentrations $(30-59 \mathrm{mmol} / \mathrm{L}$ for infants less than 6 months of age, $40-59 \mathrm{mmol} / \mathrm{L}$ for older individuals) and two CF mutations with a different degree of CFTR protein dysfunction [14]. Finally, the growing proportion of NBS programs revealed a large number of infants having a Cystic Fibrosis Screen Positive, Inconclusive Diagnosis (CFSPID) in Europe or CFTR - related metabolic syndrome (CRMS) in the US including those infants with elevated IRT levels, but with insufficient sweat chloride or genetic data to support a diagnosis of CF $[15,16]$.

Nowadays the use of CFTR gene sequencing [17] led to the detection of a number of variants for which it lacks a clear genotype-phenotype correlation. Some of these can reach a higher frequency only in certain populations, due to a founder effect in ethnic or geographical isolates $[18,19]$.
S737F (c.2210C > T; p.Ser737Phe) is a CFTR missense variant, located in exon 13 and characterized by a substitution of serine with phenylalanine in position 737, identified in 2004 in two Italian patients with pancreatic sufficiency (PS) and pathological sweat chloride level (SCL) in one case $(\mathrm{Cl} 71 \mathrm{mEq} / \mathrm{l})$ and borderline in the other (Cl $51 \mathrm{mEq} / \mathrm{l})$ (submitted to http://www.genet.sick kids.on.ca/cftr/app). Functional classification of this variant still remains unknown and no data are available in literature at the moment.

The aim of our study is to describe clinical features of patients bearing such rare variant and to assess the residual function of CFTR protein on nasal epithelial cells obtained by nasal brushing.

\section{Methods}

Patients population

We performed a retrospective analysis of clinical records of all patients either homozygous or compoundheterozygous for the S737F CFTR missense variant between 295 patients followed in the Cystic Fibrosis Centre of Tuscany region (Italy). Demographic, clinical, genetic and biochemical data regarding all enrolled patients were extracted from local electronic health records. All patients had given consent to the recording of their clinical data and for their anonymous use for scientific purposes, including descriptive studies. Furthermore, the approval of Ethics Committee of University Federico II of Naples was obtained for sampling of nasal epithelial cells and for the functional analysis of novel mutations in patients with $\mathrm{CF}$.

\section{Diagnostic tests and clinical data}

All patients underwent NBS for CF by the analysis of blood IRT at day 3 performed by using Auto-DELFIA method until 2013 and by the GSP instrument from 2014 (Perkin-Elmer). Meconium lactase was detected by glucose production after incubation of meconium with lactose. The NBS algorithm is reproduced in Fig. 1. From 2011 a pilot project was aimed to compare usual diagnostic algorithm (IRT - meconium lactase - IRT2) to a new protocol IRT-DNA.

We collected a new database including demographic data and detailed clinical or genetic data at diagnosis, as follows: gender, age at diagnosis, genotype and cause of diagnosis.

SCL, obtained according to guidelines by the Gibson and Cooke method, $[11,20]$ were analyzed at diagnosis and annually repeated in each patient with quantitative pilocarpine iontophoresis. Furthermore sweat test was performed in the same laboratory, thus ruling out the lack of harmonization between different labs [21].

We defined CFSPID infants who are asymptomatic, with hypertrypsinogenemia at birth and either: (1) 0 or 1 CFTR mutations, plus intermediate sweat chloride 


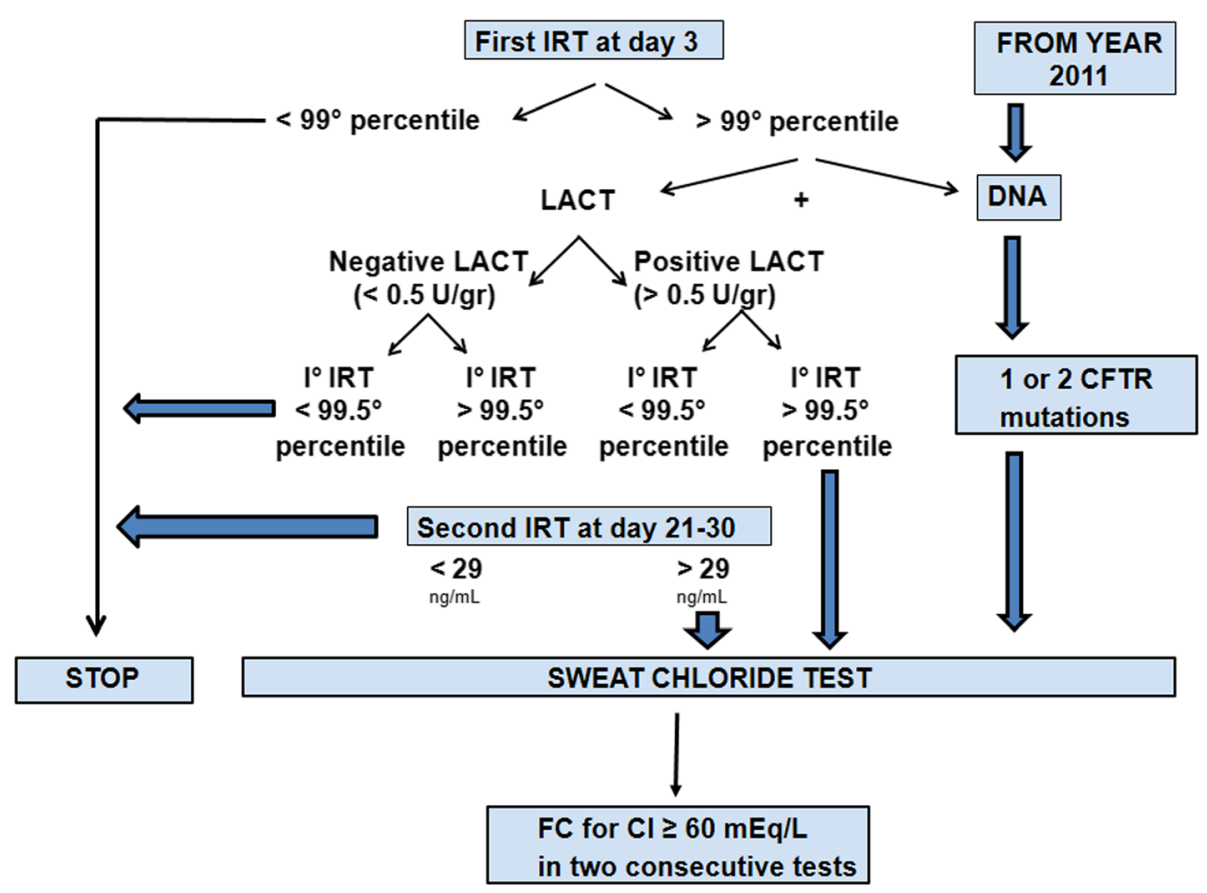

Fig. 1 The newborn screening algorithm used at the CF Centre of Florence, Italy

(30-59 mmol/L); or (2) 2 CFTR mutations, at least 1 of which has unclear phenotypic consequences, plus a normal sweat chloride $(<30 \mathrm{mmol} / \mathrm{L})$ [15].

Weight/Height pc or body mass index (BMI) pc were evaluated in patients respectively under or beyond 2 years of age [22]. Last best forced expiratory volume in the 1st second $\left(\mathrm{FEV}_{1}\right)$, expressed as percentage of predicted value for age, according to standardized reference equations for spirometry [23] were recorded for patients aged over 6 years. Given the inter-individual variability of $\mathrm{FEV}_{1}$ and the evolution of lung damage with age, the patients were classified as "severe" or "mild" according with Schluchter et al. criteria that takes into account both the $\mathrm{FEV}_{1}$ value and age [24]. Endobronchial bacterial infections were detected by sputum or throat swab cultures. Pseudomonas aeruginosa (PA), Stenotrophomonas malthophilia (SM), Haemophlus Influenzae (HI), Methicillin sensitive Staphylococcus aureus (MSSA) or Methicillin resistant Staphylococcus aureus (MRSA) chronic infection was defined following modified Leeds criteria [25]. PS was defined on the basis of at least two values of fecal pancreatic elastase higher than $200 \mu \mathrm{g} / \mathrm{g}$ measured outside acute gastrointestinal diseases [26]. Pancreatitis was defined according to the report from the international study group of pediatric pancreatitis [27]. Cystic fibrosis related diabetes mellitus (CFRD) was diagnosed according to the American Diabetes Association criteria [28]. CF-associated liver disease (CFLD) was defined when imaging (ultrasound, computed tomography, magnetic resonance) and demonstrated hepatic parenchymal abnormalities and/or portal hypertension (esophageal varices, portal-systemic collaterals, splenomegaly) in the absence of other causes [29]. Complications such as allergic bronchopulmonary aspergillosis (ABPA), meconium ileus (MI) or distal intestinal obstruction syndrome (DIOS), nasal polyposis, hemoptysis, and pneumothorax were registered.

\section{Genetic analysis}

According to European recommendations, [30] molecular analysis was performed testing a panel of the most frequent CFTR mutations followed by the search of the most frequent CFTR rearrangements [31] and sequencing of the whole coding regions of CFTR [14] once one or both mutations had not been identified. Before 2011 we performed genetic analysis in all infants with a pathological sweat chloride in order to confirm CF diagnosis, while since 2011 it has become part of the screening program (IRT-DNA) (Fig. 1).

\section{Nasal brushing}

In order to assess the residual function of CFTR protein, we performed a nasal brushing in cooperating patients bearing S737F CFTR variant and compared the results with those of $\mathrm{CF}$ patients with other genotypes followed in the pediatric Cystic Fibrosis Centre of Campania region (Italy). The informed consent was required to the patients (legal guardian for minors) before sampling, after a complete description of the aims of the study. All subjects underwent a complete ear-nose-throat 
evaluation. To test the activity of the CFTR protein, we used the halide-sensitive fluorescent system. Details are provided in a previous paper of our group [32,33].

\section{Results}

\section{Genotype, clinical data and clinical course}

Nine subjects carrying at least one S737F were identified after gene sequencing (detection rate 98\%): 8 were compound heterozygous for the S737F and one class I-II mutation (mean age: 8,9 years, range $0,5-15,8$ years), while one patient ( 8,4 years) was homozygous for the S737F (Table 1).

No detection of complex alleles has been found. 7/9 patients were diagnosed by NBS and were considered CFSPID infants having a borderline SCL and one CFTR causing mutation. In the remaining two cases (resulted negative at NBS) diagnosis was performed at the age of 10 months and 20 months respectively, after an episode of dehydration with hypochloremic metabolic alkalosis during the summer needing hospitalization. At diagnosis, one case had SCL in the pathological range $(\mathrm{Cl}$ : $71 \mathrm{mEq} / \mathrm{L}$ ) while in $8 / 9$ cases SCL was in the intermediate range for age $(\mathrm{Cl} 30-59 \mathrm{mEq} / \mathrm{L}$ for infants under the age of 6 months; $\mathrm{Cl} 40-59 \mathrm{mEq} / \mathrm{L}$ for patients over 6 months) (Table 1) [10]. During follow up SCL, annually o semesterly evaluated in each patient, gradually increased with age: the last SCL was pathological for age (range $60-121 \mathrm{mEq} / \mathrm{L}$ ) in $8 / 9$ patients (defining a CF diagnosis), while in only one case it remains unmodified in borderline range (Table 2). The mean age at diagnosis of CF was 1,5 years (range $0,2-3,8$ ).

Lung function tests were available in 6 cooperating patients: according to Schluchter et al. criteria, [24] all patients were classified as "mild" for age (Table 2).

After a mean follow up of 8,6 years (range 0,5-15,8), all patients were PS and in good nutritional status (with five of them having a $\mathrm{BMI} \geq 95^{\circ} \mathrm{pc}$ ). None of them had presented pancreatitis, CFLD, CFRD or other complications. Sputum or throat swab cultures revealed chronic infection by MSSA in 6/9 cases and occasional infection of PA, SM, or HI in the other cases (Table 2).

Respiratory exacerbations, requiring oral antibiotics prescription were uncommon in all patients over the time (only the patient n.5 required a cycle of oral ciprofloxacin and aerosol therapy in order to eradicate PA infection). Chest High Resolution Computed Tomography scan showed the absence of bronchiectasis in patients n. 5 and n. 6 . In order to exclude CBAVD presence, we proposed the analysis of spermiograms for the two males adolescents patients but the parents refused.

\section{Nasal brushing}

As shown in Fig. 2, we analyzed the CFTR gating activity on nasal epithelial cells obtained from several CF patients and carriers. In particular, we studied: i) three patients with CF of which one was homozygous for the F508del mutation (case \#1), another was compound heterozygous for the N1303 K and the [R74;V201 M;D1270] complex allele (case \#2) and the third was compound heterozygous for the $\mathrm{N} 1303 \mathrm{~K}$ and the $711+1 \mathrm{G}>\mathrm{T} \mathrm{mu}-$ tations (case \#3) (the phenotypes are showed in Table 3 ) [32]. They showed an activity of $6,2 \%, 9,8 \%$ and $5,2 \%$, respectively; ii) a CF patient compound heterozygous for the W1282X and D1152H mutation [32] (Table 3). He had an activity of $20.3 \%$ (case \#4); iii) two patients compound heterozygous for the S737F variant with the W1282X mutation (case \#5) and with the DelE22_24 (case \#6). Such cases showed an activity of $15.6 \%$ and $12.7 \%$, respectively (the phenotypes are showed in Table 2 and in Table 3, cases \# 5 and 6); iv) two carrier subjects, heterozygous for the F508del mutation (case \#7) and for the G542X mutation (case \#8); they had an activity of 40.7 and $76,8 \%$ (Table 3 ).

\section{Discussion}

We described the first cohort of patients bearing the S737F CFTR missense variant and evaluated the residual function of CFTR protein on nasal epithelial cells

Table 1 demographic data of the enrolled patients

\begin{tabular}{lllllll}
\hline Patient & Gender & Age at diagnosis (months) & Cause of diagnosis & Second mutation & SCL at diagnosis & IRT $^{\text {a }}\left(\mathrm{ng} / \mathrm{ml}^{\prime}\right)$ \\
\hline 1 & M & 1 & NBS & F508del & 46 & 74 \\
2 & M & 20 & Hypochloremic Alkalosis & S737F & 45 & 31 \\
3 & M & 1 & NBS & F508del & 35 & 76 \\
4 & F & 1 & NBS & 541delC & 48 & 71 \\
5 & M & 10 & Hypochloremic Alkalosis & $22,23,24$ del & 71 & 37 \\
6 & M & 1 & NBS & W1282X & 51 & 79 \\
7 & F & 1 & NBS & F508del & 52 & 70 \\
8 & F & 1 & NBS & F508del & 49 & 76 \\
9 & M & 1 & NBS & F508del & 51 & 76
\end{tabular}

$S C L$ sweat chloride level, IRT blood immunoreactive trypsinogen, NBS newborn screening

a IRT is elevated for values $>99$ th centile, variable every 3-4 months from 50 to $64 \mathrm{ng} / \mathrm{ml}$ 
Table 2 Clinical data of the enrolled patients

\begin{tabular}{llllllll}
\hline Patient & Age (years) & PS & FEV & Sputum pathogens & Pc BMI ( $\left.{ }^{\circ}\right)$ & SCL at enrolment (mEq/l) & Follow up (years) \\
\hline 1 & 9,5 & Yes & NA & Hi, Mssa & 95 & 46 & 9,5 \\
2 & 8,4 & Yes & 99 & Mssa & $>95$ & 98 & 6,6 \\
3 & 9,7 & Yes & 101 & Hi & 95 & 78 & 9,7 \\
4 & 8,8 & Yes & 96 & Mssa, Sm & $75-85$ & 94 & 8,8 \\
5 & 15,7 & Yes & 99 & Pa & $75-85$ & 89 & 14.9 \\
6 & 15,8 & Yes & 108 & Mssa & $50-75$ & 121 & 15,8 \\
7 & 10,7 & Yes & 114 & Mssa & $>95$ & 60 & 10,7 \\
8 & 0,5 & Yes & NA & Mssa & $>95^{\text {a }}$ & 68 & 0,5 \\
9 & 1,5 & Yes & NA & Hi & $90^{\text {a }}$ & 67 & 1,5
\end{tabular}

PS pancreatic sufficiency, FEV forced expiratory volume in the 1st second, SCL sweat chloride level, NA not available, Hi Haemophilus Influenzae, Mssa Methicillin sensitive Staphylococcus aureus, Sm Stenotrophomonas malthophilia, $\mathrm{Pa}$ Pseudomonas Aeruginosa

a Weight/Height pc were evaluated in patients under 2 years of age

obtained by nasal brushing in comparison to patients with other genotypes. Our results show that S737F is associated to hypochloremic alkalosis in childhood, mild $\mathrm{CF}$ phenotype in teenage years and a residual function of CFTR protein. In fact, despite 7 patients resulted positive to NBS, the other two were diagnosed by a single episode of dehydration with hypochloremic metabolic alkalosis. Furthermore, all 9 patients presented PS and good nutritional status, all had normal respiratory function for age and none of them developed complications during follow up. Only one patient presented an occasional isolation of $P$. aeruginosa that eradicated after antibiotic therapy. Nevertheless the mean age and the follow up of our patients are too low to exclude a severe course of the pulmonary disease in adulthood or the development of pancreatic insufficiency.

It is known that some mutations are more frequent in specific regions or ethnic groups [18, 19]. Interestingly

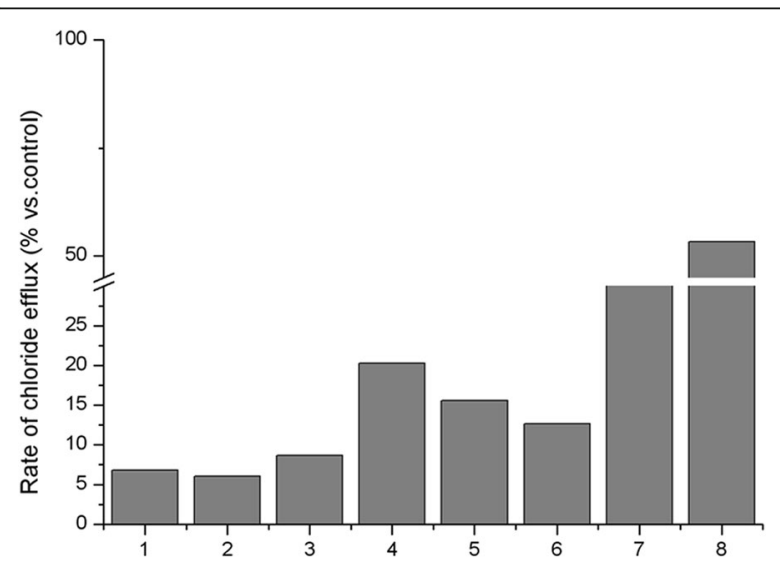

Fig. 2 CFTR gating activity on nasal epithelial cells obtained from CF patients and carriers with different genotypes. 1: F508del/F508del; 2: F508del/[R74;V201 M;D1270]; 3: N1303 K/711 + 1G > T; 4: W1282X/ D1152H; 5: S737F/W1282X; 6: S737F/DelE22_24; 7: F508del (heterozygous); 8: G542X (heterozygous) all the families of the patients were residing in the Tuscany region for at least two generations. Our data confirm the need to investigate the regional origin of each patient with suspected CF in order to reduce the residual risk that a couple negative for the test will have an affected child [19]. Furthermore is also necessary to adapt neonatal screening panels based on the frequency of mutations in each region.

The management of children with an inconclusive diagnosis following NBS has been recently discussed [15, 16]. In some cases the sweat chloride result may be in intermediate range or CFTR gene changes may be recognized, the phenotypic consequences of which are unclear. According to Munck et al. and Ooi et al., in our cohort we repeated annually or semesterly the sweat test and during follow up 6/7 CFSPID infants moved to a CF diagnosis given that sweat test moved from an intermediate to a CF-confirmatory result $[6,33,34]$. Unlike Ooi et al. [33] sweat chloride increased to abnormal levels at a slightly lower mean age (21,3 months vs 17 months).

The sensitivity of NBS for CF diagnosis depends on the IRT cut off as well as the population screened as a result of differences in the genotypic distribution for $\mathrm{CF}$ in the population, with estimates of false negative results ranging from $1.7-5.4 \%$ of children with CF [35, 36]. Delay in diagnosis of CF and, hence, delay in treatment is associated with deleterious outcomes including nutritional and growth concerns, a higher rate of complications, increased stress to the families, and the potential for poorer pulmonary outcomes [37-39]. Nevertheless, the effect of age of diagnosis on survival with CF is moot given that it is not the only factor affecting the survival $[36,40-42]$. In a 30 year retrospective review of survival with CF from two regions in Italy including diagnosis by symptoms and NBS, Assael et al. reported that patients diagnosed after 5 years of age appeared to have a survival advantage over those 
Table 3 Clinical data and CFTR gating activity on nasal epithelial cells obtained from several CF patients and carriers

\begin{tabular}{|c|c|c|c|c|c|c|c|}
\hline Patient & Age (years) & Genoytpe & $\mathrm{SCL}$ at diagnosis (mEq/L) & PS & $\mathrm{FEV}_{1}$ at enrollment (\%) & Sputum Pathogens & CFTR gating (\%) \\
\hline Case \#1 & 19 & F508del/F508del & 118 & NO & 64 & $\mathrm{~Pa}$ & 6,2 \\
\hline Case \#2 & 21 & N1303 K [R74;V201 M;D1270] & 109 & YES & 119 & Hi, Mssa & 9,8 \\
\hline Case \#3 & 23 & $\mathrm{~N} 1303 \mathrm{~K} 711+1 \mathrm{G}>\mathrm{T}$ & 89 & NO & 54 & $\mathrm{~Pa}$ & 5,2 \\
\hline Case \#4 & 19 & W1282X/D1152H & 52 & YES & 115 & Mssa & 20,3 \\
\hline Case \#5 & 15,7 & S737F/W1282X & 71 & YES & 99 & $\mathrm{~Pa}$ & 15,6 \\
\hline Case \#6 & 15,8 & S737F/Dele22_24 & 51 & YES & 108 & Mssa & 12,7 \\
\hline Case \#7 & 42 & F508del heterozygous & NA & NA & NA & NA & 40,7 \\
\hline Case \#8 & 48 & G542X heterozygous & NA & NA & NA & NA & 76,8 \\
\hline
\end{tabular}

PS pancreatic sufficiency, $F E V_{1}$ forced expiratory volume in the 1st second, SCL sweat chloride level, NA not available, Hi Haemophilus Influenzae, Mssa Methicillin sensitive Staphylococcus aureus, Pa Pseudomonas Aeruginosa

diagnosed under 1 year of age without a significant effect of NBS on survival [40].

In our cohort two patients (cases 2 and 5) resulted negative at NBS and were diagnosed at the age of 10 months and 20 months after an episode of dehydration with hypochloremic metabolic alkalosis during the summer. This is a relatively common and sometimes dangerous manifestation of CF in infancy; the possibility of CF diagnosis should be considered in any infant with this metabolic disorder even if resulted negative at newborn screening. In order to prevent these events we have included S737F mutation in cystic fibrosis neonatal panels screening and we suggest to look for S737F in subjects of Tuscany region origin with symptoms compatible with cystic fibrosis.

The analysis of CFTR gating activity on ENC confirms that such analysis may help to define the residual activity of the mutated protein and seems to correlate with the severity of the CF phenotype. In fact, the three patients with severe $\mathrm{CF}$ and compound heterozygous for two CFTR severe mutations (cases 1 to 3 ) show a very low residual CFTR activity (i.e., < 10\%); the two heterozygous subjects show an activity between 40 and $80 \%$, and the patient with mild CF, compound heterozygous for the W1282X and the mild D1152H mutation [43] had an activity in the range 10 to $20 \%$. The two cooperating patients bearing the S737F mutation show a residual CFTR activity of 12.7 and $15.6 \%$, according to a mild CF clinical expression (that seems to be associated to residual activity ranging from 10 to $20 \%$ ).

\section{Conclusions}

S737F seems to be a CFTR mutation, typical of patients originally from the Tuscany region, associated to a residual activity of the CFTR protein assessed in nasal epithelial cells. We have included this mutation in cystic fibrosis neonatal panels screening in order to prevent dangerous episodes of dehydration with hypochloremic metabolic alkalosis.

\section{Abbreviations}

ABPA: Allergic bronchopulmonary aspergillosis; BMI: Body mass index; CF: Cystic fibrosis; CFLD: CF liver disease; CFRD: CF related diabetes; CFSPID: Cystic Fibrosis Screen Positive, Inconclusive Diagnosis; CFTR: Cystic fibrosis transmembrane regulator; CFTR-RD: CFTR-related disorder; CRMS: CFTR-Related Metabolic Syndrome; DIOS: Distal intestine obstructive syndrome; HI: Haemophilus Influenzae; IRT: Immunoreactive trypsinogen; MI: Meconium ileus; MRSA: Methicillin resistant Staphylococcus aureus; MSSA: Methicillin sensitive Staphylococcus aureus; NBS: Newborn screening; NEC: Nasal epithelial cells; PA: Pseudomonas aeruginosa; PI: Pancreatic insufficiency; PS: Pancreatic sufficiency; SCL: Sweat chloride level;

SM: Stenotrophomonas malthophilia

\section{Acknowledgements}

Not applicable

Funding

No funding was received.

Availability of data and materials

All data and material are available.

\section{Authors' contributions}

V.T., G.C and C.B conceived and designed the study, collected the data and wrote the manuscript; AM. DL. And M.C performed the nasal brushing and analysed CFTR channel activity; C.C and E.P. detected the new mutation; VR provided patient data followed in the pediatric Cystic Fibrosis Center of Naples and revised the paper. All authors approved its submission.

\section{Ethics approval and consent to participate}

The approval of Ethics Committee of University Federico II of Naples was obtained.

\section{Consent for publication}

All patients had given consent to the recording of their clinical data and for their anonymous use for scientific purposes, including descriptive studies.

\section{Competing interests}

The authors declare that they have no competing interests.

\section{Publisher's Note}

Springer Nature remains neutral with regard to jurisdictional claims in published maps and institutional affiliations.

\section{Author details}

${ }^{1}$ Centro Regionale Toscano per la Fibrosi Cistica, Azienda Ospedaliero-Universitaria Meyer, Via Gaetano Pieraccini 24, 50141 Florence, Italy. ${ }^{2}$ CEINGE-Biotecnologie Avanzate scarl, Naples, Italy. ${ }^{3}$ Dipartimento di Medicina Molecolare e Biotecnologie Mediche, Università di Napoli Federico II, Naples, Italy. ${ }^{4}$ Dipartimento di Neuroscienze, Sezione di ORL, Università di Napoli Federico II, Naples, Italy. ${ }^{5}$ SOD Diagnostica Genetica, Azienda Ospedaliera Universitaria Careggi, Florence, Italy. ${ }^{6}$ Centro Regionale Fibrosi 
Cistica Unità Pediatrica, Dipartimento di Scienze Mediche Traslazionali, Università di Napoli Federico II, Naples, Italy.

\section{Received: 26 April 2017 Accepted: 26 December 2017 Published online: 03 January 2018}

\section{References}

1. Farrell PM, White TB, Ren CL, et al. Diagnosis of cystic fibrosis: consensus guidelines from the Cystic Fibrosis Foundation. J Pediatr. 2017;181S:S4-S15.e1.

2. Bossi A, Casazza G, Padoan R, Milani S. What is the incidence of cystic fibrosis in Italy? Data from the National Registry (1988-2001). Hum Biol. 2004;76:455-67.

3. Castellani C, Cuppens H, Macek M Jr, et al. Consensus on the use and interpretation of cystic fibrosis mutation analysis in clinical practice. J Cyst Fibros. 2008;7:179-96.

4. Fanen P, Wohlhuter-Haddad A, Hinzpeter A. Genetics of cystic fibrosis: CFTR mutation classifications toward genotype-based CF therapies. Int J Biochem Cell Biol. 2014:52:94-102.

5. O'Sullivan BP, Freedman SD. Cystic fibrosis. Lancet. 2009;373:1891-904.

6. Farrell PM, Kosorok MR, Rock MJ, et al. Early diagnosis of cystic fibrosis through neonatal screening prevents severe malnutrition and improves longterm growth. Wisconsin cystic fibrosis neonatal screening study group. Pediatrics. 2001;107:1-13

7. Assael BM, Casazza G, lansa P, et al. Growth and long-term lung function in cystic fibrosis: a longitudinal study of patients diagnosed by neonatal screening. Pediatr Pulmonol. 2009:44:209-15.

8. Djick FN, Fitzgerald DA. The impact of newborn screening and earlier intervention on the clinical course of cystic fibrosis. Paediatr Respir Rev. 2012;13:220-5.

9. Barben J, Castellani C, Dankert-Roelse J, et al. The expansion and performance of national newborn screening programmes for cystic fibrosis in Europe. J Cyst Fibros. 2017;16:207-13.

10. Farrell PM, Rosenstein BJ, White TB, et al. Guidelines for diagnosis of cystic fibrosis in newborns through older adults: Cystic Fibrosis Foundation consensus report. J Pediatr. 2008:53:S4-S14.

11. Clinical and Laboratory Standard Institute (CLSI). Sweat testing: sample collection and quantitative analysis; approved guideline - third edition. CLSI document C34-A2 (ISBN 1-56238-713-8). Wayne: Clinical and Laboratory Standard Institute: 2009.

12. Bombieri C, Claustres M, De Boeck K, et al. Recommendations for the classification of diseases as CFTR-related disorders. J Cyst Fibros. 2011;10: S86-S102.

13. Terlizzi $\mathrm{V}$, Tosco A, Tomaiuolo $\mathrm{R}$, et al. Prediction of acute pancreatitis risk based on PIP score in children with cystic fibrosis. J Cyst Fibros. 2014;14:30-7.

14. Amato F, Bellia C, Cardillo G, et al. Extensive molecular analysis of patients bearing CFTR-related disorders. J Mol Diagn. 2012;14:81-9.

15. Munck A, Mayell SJ, Winters V, Shawcross A, Derichs N, Parad R, et al. Cystic fibrosis screen positive, inconclusive diagnosis (CFSPID): a new designation and management recommendations for infants with an inconclusive diagnosis following newborn screening. J Cyst Fibros. 2015;14:706-13.

16. Ren CL, Borowitz DS, Gonska T, Howenstine MS, Levy H, Massie J, Milla C, Munck A, Southern KW. Cystic fibrosis Transmembrane conductance regulator-related metabolic syndrome and cystic fibrosis screen positive, inconclusive diagnosis. J Pediatr. 2017:181S:S45-S51.e1.

17. Castaldo G, Polizzi A, Tomaiuolo R, et al. Comprehensive cystic fibrosis mutation epidemiology and haplotype characterization in southern Italy population. Ann Hum Genet. 2005;69:15-24.

18. Castaldo G, Fuccio A, Cazeneuve C, et al. Detection of five rare cystic fibrosis mutations peculiar to southern Italy: implications in screening for the disease and phenotype characterization for patients with homozygote mutations. Clin Chem. 1999:45:957-62.

19. Mateu $E$, Calafell F, Ramos MD, et al. Can a place of origin of the main cystic fibrosis mutations be identified? Am J Hum Genet. 2002;70:257-64.

20. Gibson LE, Cooke RE. A test for concentration of electrolytes in sweat in cystic fibrosis of the pancreas utilizing pilocarpine by iontophoresis. Pediatrics. 1959;23:545-9

21. Christiansen AL, Nybo M. Lack of harmonization in sweat testing for cystic fibrosis - a national survey. Scand J Clin Lab Invest. 2014;74:708-12.

22. Borowitz D, Baker RD, Stallings V. Consensus report on nutrition for pediatric patients with cystic fibrosis. J Pediatr Gastroenterol Nutr. 2002;35:246-59.
23. Quanjer PH, Stanojevic S, Cole TJ, et al. Multiethnic reference values for spirometry for the 3-95 year age range: the global lung function 2012 equations. Eur Respir J. 2012;40:1324-43.

24. Schluchter MD, Konstan MW, Drumm ML, et al. Classifying severity of cystic fibrosis lung disease using longitudinal pulmonary function data. Am J Respir Crit Care Med. 2006;174:780-6.

25. Döring $\mathrm{G}$, Flume $\mathrm{P}$, Heijerman $\mathrm{H}$, et al. Consensus study group. Treatment of lung infection in patients with cystic fibrosis: current and future strategies. J Cyst Fibros. 2012;11:461-79.

26. Loser C, Mollgaard A, Folsch UR. Faecal elastase 1: a novel, highly sensitive, and specific tubeless pancreatic function test. Gut. 1996:39:580-6.

27. Morinville VD, Husain SZ, Bai $H$, et al. Definitions of pediatric pancreatitis and survey of present clinical practices. J Pediatr Gastroenterol Nutr. 2012; 55:261-5

28. Kelly A, Moran A. Update on cystic fibrosis-related diabetes. J Cyst Fibros. 2013;12:318-31.

29. Debray D, Kelly D, Houwen $R$, et al. Best practice guidance for the diagnosis and management of cystic fibrosis-associated liver disease. J Cyst Fibros. 2011;10:S29-36

30. Dequeker E, Stuhrmann M, Morris MA, et al. Best practice guidelines for molecular genetic diagnosis of cystic fibrosis and CFTR-related disorders: updated European recommendations. Eur J Hum Genet. 2009:17:51-65.

31. Tomaiuolo R, Sangiuolo F, Bombieri C, et al. Epidemiology and a novel procedure for large scale analysis of CFTR rearrangements in classic and atypical CF patients: a multicentric Italian study. J Cyst Fibros. 2008;7:347-51.

32. Terlizzi V, Castaldo G, Salvatore D, et al. Genotype-phenotype correlation and functional studies in patients with cystic fibrosis bearing CFTR complex alleles. J Med Genet. 2017;54:224-35.

33. Di Lullo AM, Scorza M, Amato F, Comegna M, Raia V, Maiuri L, llardi G, Cantone $\mathrm{E}$, Castaldo G, lengo M. An "ex vivo model" contributing to the diagnosis and evaluation of new drugs in cystic fibrosis. Acta Otorhinolaryngol Ital. 2017;37(3):207-13.

34. Ooi CY, Castellani C, Keenan $\mathrm{K}$, et al. Inconclusive diagnosis of cystic fibrosis after newborn screening. Pediatrics. 2015;135:e1377-85.

35. Munck A, Delmas D, Audrézet MP, Lemonnier L, Cheillan D, Roussey M Optimization of the French cystic fibrosis newborn screening programme by a centralized tracking process. J Med Screen. 2017:969141317692611. https://doi.org/10.1177/0969141317692611.

36. Munck A, Dhondt JL, Sahler C, et al. Implementation of the French nationwide cystic fibrosis newborn screening program. J Pediatr. 2008;153: 228-33.

37. Maclean JE, Solomon M, Corey M, et al. Cystic fibrosis newborn screening does not delay the identification of cystic fibrosis in children with negative results. J Cyst Fibros. 2011:10:333-7.

38. Farrell PM, Lai HJ, Li Z, et al. Evidence on improved outcomes with early diagnosis of cystic fibrosis through neonatal screening: enough is enough! J Pediatr. 2005;147:S30-S366.

39. Farrell PM, Li Z, Kosorok MR, et al. Bronchopulmonary disease in children with cystic fibrosis after early or delayed diagnosis. Am J Respir Crit Care Med. 2003;168:1100-8.

40. Mastella G, Zanolla L, Castellani C, et al. Neonatal screening for cystic fibrosis: long-term clinical balance. Pancreatology. 2001;1:531-7.

41. Assael BM, Castellani C, Ocampo MB, et al. Epidemiology and survival analysis of cystic fibrosis in an area of intense neonatal screening over 30 years. Am J Epidemiol. 2002;156:397-401.

42. Lai HJ, Cheng Y, Farrell PM. The survival advantage of patients with cystic fibrosis diagnosed through neonatal screening: evidence from the United States Cystic Fibrosis Foundation registry data. J Pediatr. 2005;147:S57-63.

43. Terlizzi V, Carnovale V, Castaldo G, et al. Clinical expression of patients with the D1152H CFTR mutation. J Cyst Fibros. 2015;14:447-52. 